\title{
Optic Neurodegeneration: Time to Act
}

\section{Nuri Gueven*}

Pharmacy, School of Medicine, Faculty of Health, University of Tasmania, Hobart, TAS, Australia

*Corresponding author: Nuri Gueven, Pharmacy, School of Medicine, Faculty of Health, University of Tasmania, Hobart, TAS, Australia, Tel: +61 3 6226 171; E-mail: nguven@utas.edu.au

Received date: May 21, 2014; Accepted date: July 10, 2014; Published date: July 17, 2014

Copyright: @ 2014 Nuri G, et al. This is an open-access article distributed under the terms of the Creative Commons Attribution License, which permits unrestricted use, distribution and reproduction in any medium, provided the original author and source are credited.

\begin{abstract}
Optic neuropathies are frequently associated with mitochondrial dysfunction and the associated vision loss has a severe impact on the patient's quality of life. Our understanding of disease progression of one of the most frequent mitochondrial disorders, Leber's hereditary optic neuropathy (LHON), together with results of recent clinical trials might provide us with new insights that are relevant not only to the progression of the disease but more importantly also for therapeutic intervention. One of the crucial hallmarks of LHON is the occasional recovery of vision in some patients. This rare and spontaneous process highlights that blindness in LHON patients is not irreversible per se and suggests that this process could potentially be induced by pharmacological intervention. Strikingly, spontaneous recovery of vision has been reported in some patients several years after disease onset, which indicates the presence of an extended time window where recovery is still possible, before over time the terminal loss of retinal neurons renders visual recovery impossible. Several recent encouraging trials in LHON and related disorders support this view and extend this model to other optic neuropathies that are not associated with spontaneous recovery. This concept provides hope not only to mitochondrial optic neuropathy patients, but also to patients that suffer from one of the major ocular disorders such as glaucoma.
\end{abstract}

\section{LHON as Example of a Neurodegenerative Retinal Disease}

Among many of the known neurodegenerative diseases, disorders that affect vision are ideally suited to test potential therapeutic interventions. This advantage is a direct consequence of the possibility to monitor and quantify degenerative processes in a timely and noninvasive manner, using standardized tests to easily quantify visual acuity and retinal pathology. In their own right, optic neuropathies are a major public health issue with glaucoma being one of the most prominent disorders. However, a relatively rare disorder, Leber's hereditary optic neuropathy (LHON; OMIM 535000), might serve as a promising model system for the experimental analysis of a range of optic nerve diseases. LHON was first described by German ophthalmologist Theodor Leber in 1871 as a distinct clinical entity [1]. For North-East England, a minimum prevalence of 1 in 31000 was estimated for LHON [2] and a recent meta-analysis suggests a prevalence of 1 in 48000 across Europe [3]. LHON is characterized by acute or sub-acute vision loss in one eye, generally followed by loss of visual acuity in the second eye within 2 to 4 months [4,5]. Initially, LHON patients experience painless vision loss, which is severe and associated with dense central or centrocecal scotoma and impaired colour vision. Typically, LHON affects young adult males of all ethnic groups, with a peak of onset in young adulthood. While in most cases vision loss is permanent, a minority of patients show spontaneous recovery of visual acuity $[4,6]$ by a mechanism that is not yet understood. Consequently, for the vast majority of young patients the diagnosis of LHON is associated with a significant reduction in their quality of life, even when compared to other ophthalmic disorders [7].

LHON is a mitochondrial disease that is caused by well characterized mutations in mitochondrial DNA (mtDNA), with a prevalence of carriers of about 1 in 300 [8]. In 95\% of all cases, LHON is caused by one of three point mutations in subunits of complex I:
3460G $>\mathrm{A}$ in MTND1, 11778G $>\mathrm{A}$ in MTND4, and $14484 \mathrm{~T}>\mathrm{C}$ in MTND6 [8]. The 11778G $>$ A mutation is generally the most abundant, although there is considerable variation in the relative frequency of the three primary LHON mutations worldwide. In addition to the primary mtDNA mutations and the overall load of mutants versus wild-type mtDNA, the individual genetic mitochondrial haplotype also plays a major role for disease onset and severity [9]. A significantly increased risk of vision loss was observed when the $11778 \mathrm{G}>\mathrm{A}$ and $14484 \mathrm{~T}>\mathrm{C}$ mutations were present in a carrier with mtDNA haplogroup $\mathrm{J}$ background. On the other hand, 3460G $>$ A carriers were more likely to lose vision when also belonging to the mtDNA haplogroup $\mathrm{K}$. In contrast, haplogroup $\mathrm{H}$ was associated with a protective effect in combination with the $11778 \mathrm{G}>\mathrm{A}$ mutation [9].

\section{Biochemical defects in LHON cells}

It is striking that all of the three main primary LHON mutations result in amino acid changes in subunits of complex I (NADH:ubiquinone oxidoreductase) of the mitochondrial respiratory chain. Experimental evidence strongly connects complex I dysfunction to reduced mitochondrial membrane potential, decreased ATP synthesis and elevated levels of oxidative stress. Consistent with the $11778 \mathrm{G}>\mathrm{A}$ mutation being associated with the most severe disease phenotype, this mutation is also associated with the most pronounced reduction in mitochondrial ATP synthesis followed by the $14484 \mathrm{~T}>\mathrm{C}$ and then the $3460 \mathrm{G}>\mathrm{A}$ mutation [10-12]. However, it is important to point out that with regards to reduced ATP synthesis no major differences between LHON patients and unaffected carriers have been demonstrated so far. This entirely mutation-dependent reduction in energy supply is supported by studies using cybrids carrying LHON mutations as well as peripheral lymphocytes from patients and mutation carriers [13]. 
In addition to reduced energy output, impaired mitochondrial electron flow is well known to generate elevated levels of reactive oxygen species (ROS). In line with this defect, elevated levels of ROS were also observed in LHON cybrids in vitro, harboring any of the three primary LHON mutations [14-17]. Consistent with these in vitro data, elevated biomarkers of oxidative stress were also found in blood leucocytes of LHON patients $[18,19]$. It is thought that elevated ROS levels are also responsible for a glutamate transport defect observed in LOHN cybrids, since antioxidants were able to correct this deficiency [20]. In the acute phase of the disease, which can last for prolonged periods of time (years rather than weeks), this reduced energy production combined with elevated levels of ROS is thought to impair the primary function of retinal ganglion cells (RGC), which is to transmit optical signals to the brain. Over time, during the atrophic phase, elevated ROS levels and as a secondary event, glutamate excitoxicity [21], which are both potent inducers of cell death, lead RGCs to die by a specific form of apoptosis that is thought to be Fasdependent but caspase independent [22,23]. This apoptotic loss of $\mathrm{RGC}$ is regarded as the reason for the final and permanent loss of vision (Figure 1).

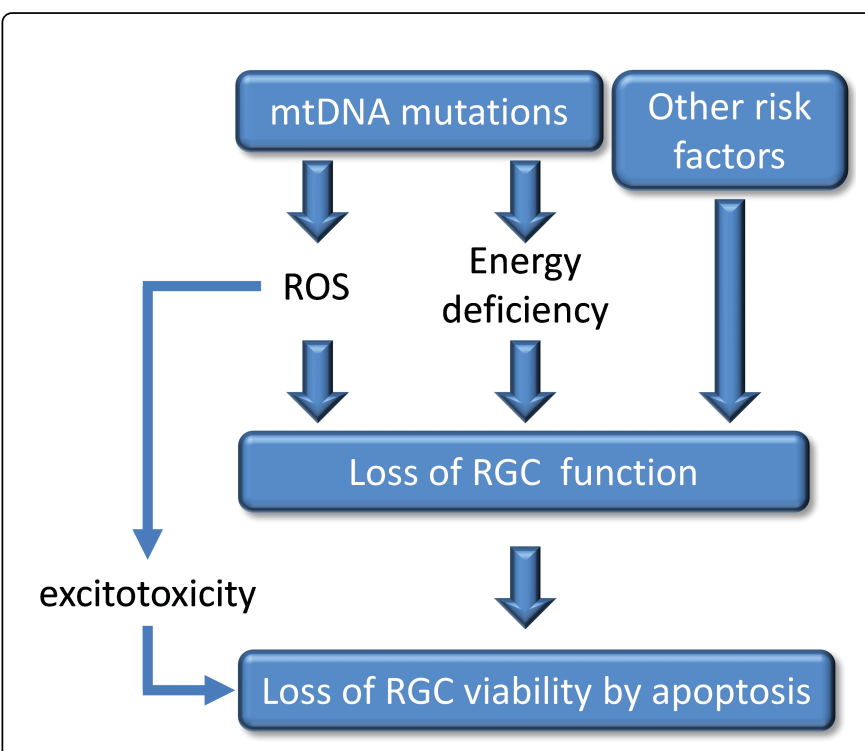

Figure 1: Molecular pathology of LHON RGC; Mitochondrial DNA mutations, which are responsible for impaired energy production and excess ROS, have to act in concert with other risk factors to impair RGC function. This RGC dysfunction eventually leads to RGC loss by an apoptotic cell death mechanism.

\section{Why are RGCs especially vulnerable?}

It is important to point out that in LHON patients mtDNA mutations and the associated mitochondrial dysfunction are prevalent in all cells including lymphocytes [13]. However, despite some peripheral neuropathy, tremor, CNS signs and rare cases of LHONmultiple sclerosis-overlap syndrome [24-27], the disease pathology is largely restricted to the RGC. Although, no unambiguous reason for this selective vulnerability has been identified, it was proposed that it arises as consequence of the unique structure of RGC within the retina and the optic nerve [28,29]. Overall, given the high energy requirements of the Central Nervous System (CNS) compared to other tissues, it is not surprising that a dysfunction of energy production would affect neurons of the CNS. At the same time, it has to be noted that this high energy expenditure of the CNS has already been optimized by nature by the invention of axonal myelin sheeting. The use of myelin significantly saves cellular energy by allowing the saltatory conduction of signals along the axon, where the de-and subsequent repolarization of the axonal membrane is restricted to the nodes of Ranvier [30] (Figure 2). Unlike CNS neurons however, the intraocular component of the RGC fibers are not myelinated. Only after passing through the lamina cribrosa, do the RGC-derived fibers acquire myelin sheeting by oligodendrocytes (Figure 2). In line with this structural distinction, significantly higher numbers of mitochondria were observed in the un-myelinated, intraocular part of the axon, while in the optic nerve lower numbers of mitochondria are mostly restricted to the nodes of Ranvier (Figure 2), representing areas of different energy requirements [31]. Consequently, the unique absence of myelination of the intraocular part of RGC axons, together with the resulting much higher energy demand in this area, could well be the reason for the selective vulnerability of RGC to impaired energy supply. The hypothesis that the retinal part of the axon is important for the neurodegeneration seen in LHON is also supported by the regional increased thickness of the retinal nerve fibre layer (RNFL), which is observed early on in disease development [32].

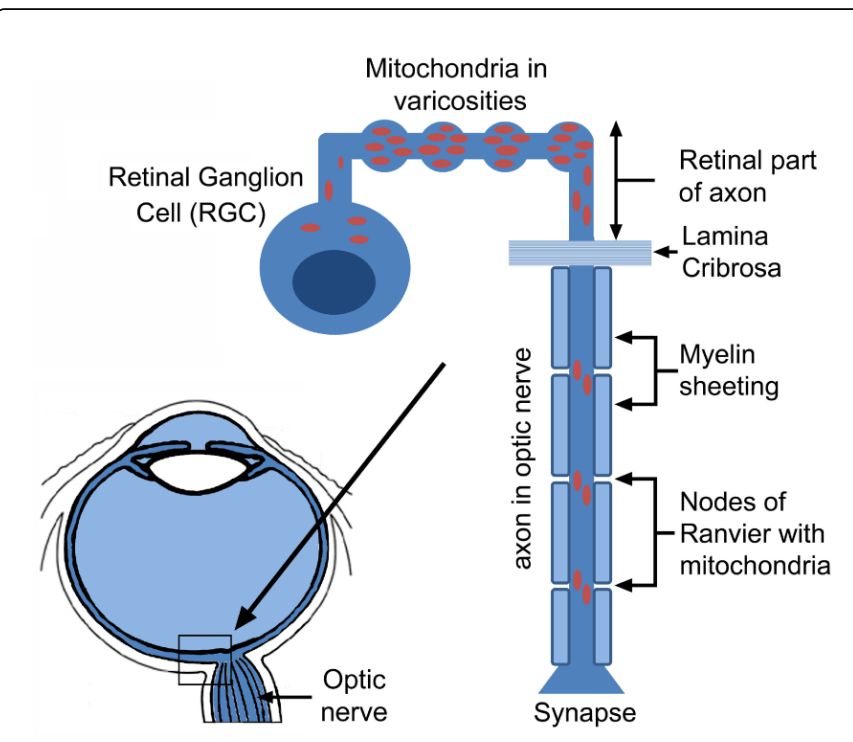

Figure 2: Structural determinant of selective RGC vulnerability in LHON; Differential distribution of mitochondria (red) of the RGC axon in the retina (pre-laminar) and within the optic nerve (postlaminar). Significantly higher mitochondrial numbers are present in the pre-laminar region as opposed to the post-laminar regions, where mitochondria are largely restricted to the Nodes of Ranvier to energize salutatory electrical signal transduction (adapted from $[6])$.

\section{Spontaneous recovery of visual acuity in LHON}

It is important to point out that LHON differs from other related mitochondrial optic neuropathies, such as dominant optic atrophy (DOA), since in a small portion of patients spontaneous recovery of vision was described. Indeed, in those patients, particular during the first year after disease onset, vision can improve to near normality of 20/40 [33], which normally manifest itself as small islands that can 
support scanning vision [8]. Strikingly however, in some patients, recovery of near normal vision in at least one eye has been described even several years after disease onset [30]. Although recovery rates have been estimated at only $4 \%$ for the $11778 \mathrm{G}>\mathrm{A}$ mutation [31], recovery rates in general are dependent on the underlying LHON mutation. While patients harboring the $11778 \mathrm{G}>\mathrm{A}$ mutation display the lowest recovery rates, patients with the $14484 \mathrm{~T}>\mathrm{C}$ mutation have the highest chance of visual recovery, with the $3460 \mathrm{G}>$ mutation being intermediate between the two $[34,35]$. Given the molecular defects and the etiology of LHON described above, the question arises why recovery of vision, as observed in some patients, is possible at all? Surely, RGC loss has to be seen as a terminal endpoint that could only be overcome by de-novo neurogenesis, a process, which is highly unlikely in the adult human retina. On the other hand, it is conceivable that a better understanding of this naturally occurring mechanism of visual recovery could indeed serve as a guide to therapeutically influence the pathology of LHON and might even be applicable to other related diseases. Most of the currently available data point towards a hypothesis that could explain this phenomenon, which was initially postulated by Howell [36]. Howell highlighted the importance to distinguish between the initial loss of RGC/optic nerve-function, as opposed to the late stage RGC death and optic nerve degeneration, which included the formal division of disease progression of LHON patients into three separate stages: the pre-symptomatic phase, the acute phase and the atropic phase (Figure 3). Although, visual acuity is unaffected in pre-symptomatic carriers of LHON mutations, this phase is already characterized by peripapillary microangiopathy [1], which involves tortuous vessels in the central retina and telangiectatic capillaries. The combination of LHON mutations with the presence of unidentified, secondary, genetic or environmental factors will initiate the acute phase of the disease (Figure 3). This phase is first and foremost characterized by a loss of visual acuity that is associated with all the cellular pathologies such as swelling of the retinal nerve cell layer, energy deficiency and oxidative stress and as a consequence, dysfunctional RGC that, despite being alive, are unable to transmit the optical information to the brain. Only in the final, atrophic phase are RGC terminally lost and the degeneration of the optic nerve becomes evident (Figure 3). It is obvious that in this terminal phase of LOHN, visual recovery appears very unlikely. On the other hand, the presence of dysfunctional RGC in the acute phase opens up a realistic possibility of rescuing and restoring vision, as illustrated by the reported cases of spontaneous recovery. Based on this hypothesis, it is of course of extreme interest to understand the time periods for the acute phase that can be expected in LHON patients, since this time interval would represent a window of opportunity in which recovery of vision is possible. Disease progression will certainly differ from patient to patient depending on the underlying mutation and the presence of additional factors. However, based on the available information of spontaneous recovery, in some patients, time periods of up to several years are not unrealistic for the acute phase [33]. This potentially large window of opportunity is an encouraging concept for our ability to therapeutically restore vision in these patients.

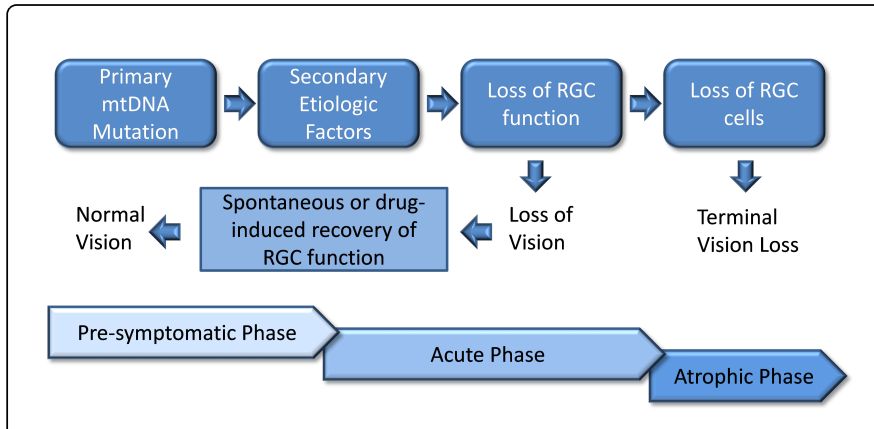

Figure 3: Natural history of LHON; Loss of vision during the acute phase of LHON is thought to be a result of a loss of RGC function, whereas during the atrophic phase this loss of vision is made irreversible by a terminal loss of RGC together with a degeneration of the optic nerve. During the acute phase of LHON, the possibility for natural recovery of vision highlights that pharmacological interference at this time with the aim to restore RGC function could also rescue visual acuity (adapted from [36]).

\section{Treatment options}

Several strategies and compounds have been tested in pre-clinical model systems and a limited number of clinical trials to alleviate the pathology associated with LHON have been reviewed previously [37]. The currently investigated approaches range from pharmacological intervention to physical therapy and gene therapy. Gene therapy aims to restore the underlying defect of each individual patient by ectopic expression of the defective mitochondrial subunit and could therefore by a curative approach. However, despite encouraging-preclinical reports [38] and ongoing trials [37] this method is still unproven and it should not be ignored that some criticism of this approach has been voiced $[39,40]$. Therefore, only the results of the ongoing and future clinical trials will indicate if this approach will be effective. Furthermore a single attempt at testing a physical therapy for LHON was conducted (NCT01389817) that aimed to test if near-infrared therapy might stimulate mitochondrial function, attenuate oxidative stress, and/or improve cell survival. This trial was initiated based on encouraging neuroprotective data from preclinical studies of other indications [41]. Unfortunately, the trial was terminated early as the patients were unable to focus sufficiently for the primary measure. Overall, the majority of studies aimed at identifying pharmacological interventions to improve vision in LHON patients, although with mixed success [37].

\section{How can a therapeutic strategy be rationalized?}

LHON is characterized by incomplete penetrance, with only about $50 \%$ of males and $10 \%$ of female carriers actually loosing visual acuity [24]. These epidemiological observations, together with experimental data using cells from carriers and LHON patients, indicate that energy deficiency, elevated levels of ROS and apoptosis susceptibility are not solely responsible for the development of LHON but act in conjunction with secondary genetic, epigenetic or environmental factors. However, it is clear that energy deficiency and oxidative stress are major contributing factors, either directly or indirectly, for example by influencing additional pathologies such as axonal transport and excitotoxicity that lead to cell death. Given their importance, it can be speculated that compounds that can reduce the 
oxidative burden and/or improve energy supply in RGC should therefore be of therapeutic value. In fact, supportive evidence for the concept of quiescent, dysfunctional retinal neurons due to limited energy supply could come from a recent placebo- controlled, randomized trial involving patients with non-diabetic open-angle glaucoma [42]. Patients were given either saline or eye drops containing $50 \%$ glucose before contrast sensitivity and best-corrected logarithm of the minimum angle of resolution (logMAR) were evaluated. Overall, glucose treatment significantly improved contrast sensitivity compared to saline treatment by $0.26 \log$ units $(95 \%$ confidence interval $[\mathrm{CI}], 0.13-0.38 ; \mathrm{P}<0.001)$ and $0.40 \log$ units $(95 \%$ CI, 0.17-0.60; $\mathrm{P}<0.001)$ in a follow-up study. Importantly, this glucoseinduced, temporary improvement of visual parameters was not associated with changes in intraocular pressure, refraction or central corneal thickness. This observation led the authors to speculate that energy substrate delivery could be responsible for the functional recovery of "sick" retinal neurons [42]. Given the strong similarity between LHON and glaucoma with respect to disease pathology, affected cells and dysfunction of mitochondrial complex I in both diseases [43], this study supports the idea that an improved energy supply could alleviate the dysfunctional state of RGC in LHON before these cells are irreversibly lost. Several strategies to achieve improved energy production in LOHN RGC could be envisaged. As rationalized in the clinical study above [42], an increased supply of energy rich substrates could indeed facilitate higher production of chemical energy but it can be expected that these effects are likely only functional in the short term before cells adapt to this glucose oversupply, for example by down regulating the endothelial and/or retinal glucose transporter [44]. Given what we know about mitochondrial dysfunction in LHON and in particular the elevated ROS levels, it is unlikely that an increased oxygen supply could be of much benefit to increase energy output in this disease. However, even if mitochondrial function is reduced in LHON, this could conceptually be overcome by simply increasing mitochondrial numbers. While this strategy has some merit in other disorders, it is questionable if this approach could work given the structural limitations of the specific cells affected in LHON. As pointed out above, in contrast to RGC axons in the optic nerve, the retinal part of the RGC is already heavily populated with mitochondria so it remains questionable, if mitochondrial mass could be further increased in this crucial compartment. In addition, it is specifically the small diameter fibers that are predominantly affected in LHON, which further suggests that increasing mitochondrial numbers might simply be not possible due to intraaxonal space limitations.

This leaves the option of improving mitochondrial function itself. However, in the light of a dysfunctional complex I in LOHN cells, attempts to increase mitochondrial productivity have to involve strategies that are independent of complex I.

\section{Short-chain Quinones}

One potential pathway to achieve a complex I-independent form of mitochondrial respiration was recently described by us and others and involves the use of short-chain benzoquinones. We observed that some of those compounds are able to partially restore mitochondrial electron flow under conditions of a dysfunctional complex I [45,46]; an observation that was later independently confirmed [47]. This mechanism is based on the repeated transfer of $\mathrm{NAD}(\mathrm{P}) \mathrm{H}$ equivalents from the cytosol into the mitochondria and is dependent on a specific cellular reductase activity [45]. Some short-chain quinones are efficiently reduced by the cytoplasmic enzyme NADH-quinone oxidoreductase 1 (NQO1), before they enter the mitochondria to become re-oxidized by complex III of the mitochondrial electron transport chain. This process directly restores electron flow from complex III to cytochrome $c$ and as a consequence, supports the mitochondrial proton gradient and ATP synthesis by circumventing complex I-dependent electron transport $[45,46]$. Because this mechanism requires the quinones to repeatedly cross membranes when shuttling between cytoplasma and mitochondrial matrix, these quinones have to meet certain physico-chemical requirements, the most important of which is a balanced lipophilicity [46]. This requirement is also the reason why related molecules with higher lipophilicity such as CoQ10 are unable to perform this function. One of the short-chain benzoquinones that can maintain this form of complex I-independent respiration is idebenone (INN: 2-(10Hydroxydecyl)-5,6-dimethoxy-3-methyl-cyclohexa-2,5-diene-1,4-

dione). Since idebenone also stimulates additional metabolic pathways that are complex and independent and that transfer NAD (P) $\mathrm{H}$ equivalents into the mitochondria, such as the glycerophosphate (G3PDH) shuttle [48], it can't be concluded that the above described NQO1-dependent mechanism is solely responsible for the beneficial effects observed in vitro and in vivo. Nevertheless, in preclinical experiments idebenone not only reduced oxidative stress, it also protected retinal cell viability and reduced lactate production under conditions of mitochondrial dysfunction. Importantly, idebenone was also able to rescue vision and normalize retinal pathology in a mouse model of LHON [49]. Given that idebenone can act as a potent antioxidant and can initiate a complex I-independent form of respiration that sustains mitochondrial energy production, the current data suggest that idebenone would be ideally suited to counteract the molecular pathologies associated with LHON.

Consistent with this hypothesis, a number of studies and trials suggested that idebenone could have a therapeutic effect in LHON patients $[50,51]$ and provided the rationale for the first randomized, placebo-controlled study in LHON (NCT01421381) [52,53]. In this trial, idebenone improved visual acuity particularly in LHON patients with recent disease onset but not in patients of the placebo group $[52,53]$. It is encouraging to note that in patients, followed up 30 months after treatment had been terminated, the protective effect of idebenone still persisted [53]. Subsequently, this protective activity was also reported in a non-randomized, retrospective trial of idebenone [54]. It is very exciting to note that the current data seem to indicate that the concept of vision loss by dysfunctional RGC, which are nevertheless in a position to be rescued, might not be restricted to LHON but could also apply to other optic neuropathies as well. Two recent reports suggest that short-chain quinones, such as idebenone, have the potential restore vision in other optic neuropathies that in contrast to LHON normally do not show spontaneous recovery of vision $[55,56]$. In a small, pilot open-label trial, seven patients with confirmed dominant optic atrophy (DOA) were treated with idebenone (270-1000 mg/day) for at least one year and the authors reported improved visual function in all patients when compared to baseline [55]. Overall, mean visual acuity improved in both eyes and five patients reported bilateral, subjective improvement of visual function. Even in the other two patients that did not report any subjective improvement, objective measurements showed improvement of some visual functions, such as visual field, visual acuity or colour discrimination [55]. Similar results were also reported for a single Wolfram syndrome patient (WS) [56]. Like in LHON and DOA, WS is thought to be caused by mitochondrial dysfunction and is also characterized by loss of visual acuity. In this patient, increasing 
concentrations of idebenone (150-450 mg/day) led to a progressive but subjective visual recovery at 6 months. In this context, it is important to note that in this patient, idebenone treatment started at least 5 years after the onset of vision loss, which could explain the absence of measurable improvement using ophthalmologic and OCT examinations [56]. Finally, in line with the concept described above that complex I-dysfunction and elevated ROS level also apply to glaucoma [43], a recent preclinical study showed that idebenone reduced oxidant-induced cell death in human optic nerve head astrocytes from glaucoma patients [57]. This protection by idebenone was associated with reduced ROS levels, a reduction of several apoptosis markers and with increased levels of the anti-apoptotic protein bcl-2. Although, this study did not measure energy levels or ATP production in these cells in response to idebenone, the authors suggested that idebenone might also be helpful in preventing the progression of glaucomatous degeneration [57].

\section{Conclusion}

Recent clinical data using short-chain quinone compounds highlight the fact that LHON and potentially other optic neuropathies are no longer untreatable diseases. However, the positive clinical effects that were observed in some patients were mainly restricted to those in the early stages of the disease. This observation strongly indicates that there is a window of opportunity that not only coincides with described cases of spontaneous recovery of vision in LHON patients but more importantly could also be used to therapeutically rescue dysfunctional RGC neurons before they are irreversibly lost. Therefore, the success of future therapies will be directly dependent on characterizing the nature of this time window, which is most certainly influenced by individual parameters, such as the underlying mutation, lifestyle factors and genetic background. Overall however, the concept of dysfunctional retinal neurons that can still be rescued from a quiescent state will give future optic neuropathy patients, their families and doctors the confidence that there is still time to act.

\section{Acknowledgements}

This work was supported by Pharmacy, School of Medicine, University of Tasmania, Hobart, Australia.

\section{References}

1. Leber $\mathrm{T}$ (1871) Ueber hereditaere und congenital angelegte Sehnervenleiden. Graefes Arch Clin Exp Ophthalmol 17: 249-291.

2. Man PYW, Griffiths PG, Brown DT, et al. (2003) The epidemiology of Leber hereditary optic neuropathy in the North East of England. Am J Hum Genet 72: 333-339.

3. Mascialino B, Leinonen $M$, Meier $T$ (2012) Meta-analysis of the prevalence of Leber hereditary optic neuropathy mtDNA mutations in Europe. Eur J Ophthalmol 22: 461-465.

4. Fraser JA, Biousse V, Newman NJ (2010) The neuro-ophthalmology of mitochondrial disease. Surv Ophthalmol 55: 299-234.

5. Yu-Wai-Man P, Griffiths PG, Hudson G, Chinnery PF (2009) Inherited mitochondrial optic neuropathies. J Med Genet 46: 145-158.

6. Carelli V, Ross-Cisneros FN, Sadun AA (2004) Mitochondrial dysfunction as a cause of optic neuropathies. Prog Retin Eye Res 23: 53-90.

7. Kirkman MA, Korsten A, Leonhardt M, Dimitriadis K, De Coo IF et al. (2009) Quality of life in patients with Leber hereditary optic neuropathy. Invest Ophthalmol Vis Sci 50: 3112-3115.
8. Yu-Wai-Man P, Griffith PG, Chinnery PF (2011) Mitochondrial optic neuropathies - Disease mechanisms and therapeutic strategies. Prog Ret Eye Res 30: 81-114.

9. Hudson G, Carelli V, Spruijt L, Gerards M, Mowbray C et al. (2007) Clinical expression of Leber hereditary optic neuropathy is affected by the mitochondrial DNA-haplogroup background. American Journal of Human Genetics 81: 228-233.

10. Barbiroli B, Montagna P, Cortelli P, Iotti S, Lodi R, et al. (1995) Defective brain and muscle energy metabolism shown by in vivo 31P magnetic resonance spectroscopy in nonaffected carriers of $11778 \mathrm{mtDNA}$ mutation. Neurology 45: 1364-1369.

11. Lodi R, Taylor DJ, Tabrizi SJ, Kumar S, Sweeney M, et al. (1997) In vivo skeletal muscle mitochondrial function in Leber's hereditary optic neuropathy assessed by $31 \mathrm{P}$ magnetic resonance spectroscopy. Ann Neurol 42: 573-579.

12. Lodi R, Carelli V, Cortelli P, Iotti S, Valentino ML, et al. (2002) Phosphorus MR spectroscopy shows a tissue specific in vivo distribution of biochemical expression of the G3460A mutation in Leber's hereditary optic neuropathy. J Neurol Neurosurg Psychiatry 72: 805-807.

13. Brown MD, Trounce IA, Jun AS, Allen JC, Wallace DC (2000) Functional analysis of lymphoblast and cybrid mitochondria containing the 3460,11778 , or 14484 Leber's hereditary optic neuropathy mitochondrial DNA mutation. J Biol Chem 275: 39831-39836.

14. Wong A, Cavelier L, Collins-Schramm HE, Seldin MF, McGrogan M, et al. (2002) Differentiation-specific effects of LHON mutations introduced into neuronal NT2 cells. Hum Mol Genet 11: 431-438.

15. Carelli V, Rugolo M, Sgarbi G, Ghelli A, Zanna C, et al. (2004) Bioenergetics shapes cellular death pathways in Leber's hereditary optic neuropathy: a model of mitochondrial neurodegeneration. Biochim Biophys Acta-Bioenergetics 1658: 172-179.

16. Baracca A, Solaini G, Sgarbi G, Lenaz G, Baruzzi A, et al. (2005) Severe impairment of complex I-driven adenosine triphosphate synthesis in leber hereditary optic neuropathy cybrids. Arch Neurol 62: 730-736.

17. Floreani M, Napoli E, Martinuzzi A, Pantano G, De Riva V, et al. (2005) Antioxidant defences in cybrids harboring mtDNA mutations associated with Leber's hereditary optic neuropathy. FEBS J 272: 1124-1135.

18. Klivenyi P, Karg E, Rozsa C, Horvath R, Komoly S, et al. (2001) alphaTocopherol/lipid ratio in blood is decreased in patients with Leber's hereditary optic neuropathy and asymptomatic carriers of the 11778 mtDNA mutation. J Neurol Neurosurg Psychiatry 70: 359-362.

19. Yen MY, Kao SH, Wang AG, Wei YH (2004) Increased 8-hydroxy-2'deoxyguanosine in leukocyte DNA in Leber's hereditary optic neuropathy. Invest Ophthalmol Vis Sci 45: 1688-1691.

20. Beretta S, Mattavelli L, Sala G, Tremolizzo L, Schapira AH, et al. (2004) Leber hereditary optic neuropathy mtDNA mutations disrupt glutamate transport in cybrid cell lines. Brain 127: 2183-2192.

21. Mehta A, Prabhakar M, Kumar P, Deshmukh R, Sharma PL (2013) Excitotoxicity: bridge to various triggers in neurodegenerative disorders. Eur J Pharmacol 698: 6-18.

22. Ghelli A, Zanna C, Porcelli AM, Schapira AH, Martinuzzi A, et al. (2003) Leber's hereditary optic neuropathy (LHON) pathogenic mutations induce mitochondrial-dependent apoptotic death in transmitochondrial cells incubated with galactose medium. J Biol Chem 278: 4145-4150.

23. Zanna C, Ghelli A, Porcelli AM, Martinuzzi A, Carelli V, et al. (2005) Caspase-independent death of Leber's hereditary optic neuropathy cybrids is driven by energetic failure and mediated by AIF and Endonuclease G. Apoptosis 10: 997-1007.

24. Riordan-Eva P, Sanders MD, Govan GG, Sweeney MG, Da Costa J, et al. (1995) The clinical features of Leber's hereditary optic neuropathy defined by the presence of a pathogenic mitochondrial DNA mutation. Brain $118: 319-337$.

25. Nikoskelainen EK, Marttila RJ, Huoponen K, Juvonen V, Lamminen T, et al. (1995) Leber's "plus": neurological abnormalities in patients with Leber's hereditary optic neuropathy. J Neurol Neurosurg Psychiatry 59: 160-164. 
26. Charlmers RM, Harding AE (1996) A case-control study of Leber's hereditary optic neuropathy. Brain $119: 1481-1486$.

27. Harding AE, Sweeney MG, Miller DH, Mumford CJ, Kellar-Wood H, et al. (1992) Occurrence of a multiple sclerosis-like illness in women who have a Leber's hereditary optic neuropathy mitochondrial DNA mutation. Brain 115 : 979-989.

28. Kirches E (2011) LHON: Mitochondrial Mutations and More. Curr Genomics 12: 44-54.

29. Sadun AA, La Morgia C, Carelli V (2013) Mitochondrial optic neuropathies: our travels from bench to bedside and back again. Clin Experiment Ophthalmol 41: 702-712.

30. Chiu SY (2011) Matching mitochondria to metabolic needs at nodes of Ranvier. Neuroscientist 17: 343-350.

31. Barron MJ, Griffiths P, Turnbull DM, Bates D, Nichols P (2004) The distributions of mitochondria and sodium channels reflect the specific energy requirements and conduction properties of the human optic nerve head. Br J Ophthalmol 88: 286-290.

32. Barboni P, Carbonelli M, Savini G, Ramos V, Carta A, et al. (2010) Natural history of Leber's hereditary optic neuropathy: longitudinal analysis of the retinal nerve fiber layer by optical coherence tomography. Ophthalmology 117: 623-627.

33. Stone EM, Newman NJ, Miller NR, Johns DR, Lott MT, et al. (1992) Visual recovery in patients with Leber's hereditary optic neuropathy and the 11778 mutation. J Clin Neuroophthalmol 12: 10-14.

34. Harding AE, Riordan-Eva P, Govan GG (1995) Mitochondrial DNA diseases: genotype and phenotype in Leber's hereditary optic neuropathy. Muscle Nerve Suppl 3: S82-84.

35. Yu-Wai-Man P, Griffiths PG, Hudson G, Chinnery PF (2009) Inherited mitochondrial optic neuropathies. J Med Genet 46: 145-158.

36. Howell N (1998) Leber hereditary optic neuropathy: respiratory chain dysfunction and degeneration of the optic nerve. Vision Res 38 1495-1504.

37. Gueven N, Faldu D (2013) Therapeutic strategies for Leber's hereditary optic neuropathy: a current update. Intr Rare Dis Res 2: 130-135.

38. Koilkonda RD, Chou TH, Porciatti V, Hauswirth WW, Guy J (2010) Induction of rapid and highly efficient expression of the human ND4 complex I subunit in the mouse visual system by self-complementary adeno-associated virus. Arch Ophthalmol 128: 876-883.

39. Oca-Cossio J, Kenyon L, Hao H, Moraes CT (2003) Limitations of allotopic expression of mitochondrial genes in mammalian cells. Genetics 165: 707-720.

40. Perales-Clemente E, Fernández-Silva P, Acín-Pérez R, Pérez-Martos A, Enríquez JA (2011) Allotopic expression of mitochondrial-encoded genes in mammals: achieved goal, undemonstrated mechanism or impossible task? Nucleic Acids Res 39: 225-234.

41. Desmet KD, Paz DA, Corry JJ, Eells JT, Wong-Riley MT, et al. (2006) Clinical and experimental applications of NIR-LED photobiomodulation. Photomed Laser Surg 24: 121-128

42. Casson RJ, Han G2, Ebneter A2, Chidlow G2, Glihotra J2, et al. (2014) Glucose-induced temporary visual recovery in primary open-angle glaucoma: a double-blind, randomized study. Ophthalmology 121: 1203-1211.
43. Lee S, Sheck L, Crowston JG, Van Bergen NJ, O'Neill EC, et al. (2012) Impaired complex-I-linked respiration and ATP synthesis in primary open-angle glaucoma patient lymphoblasts. Invest Ophthalmol Vis Sci 53: 2431-2437.

44. Fernandes R, Carvalho AL, Kumagai A, Seica R, Hosoya K, et al. (2004) Downregulation of retinal GLUT1 in diabetes by ubiquitinylation. Mol Vis 10: 618-628.

45. Haefeli RH, Erb M, Gemperli AC, Robay D, Courdier Fruh I, et al. (2011) NQO1-dependent redox cycling of idebenone: effects on cellular redox potential and energy levels. PLoS One 6: el7963.

46. Erb M, Hoffmann-Enger B, Deppe H, Soeberdt M, Haefeli RH, et al. (2012) Features of Idebenone and Related Short-Chain Quinones that Rescue ATP Levels under Conditions of Impaired Mitochondrial Complex I. PLoS ONE 7: e36153.

47. Giorgio V, Petronilli V, Ghelli A, Carelli V, Rugolo M, et al. (2012) The effects of idebenone on mitochondrial bioenergetics. Biochim Biophys Acta 1817: 363-369.

48. Rauchová H, Drahota Z, Bergamini C, Fato R, Lenaz G (2008) Modification of respiratory-chain enzyme activities in brown adipose tissue mitochondria by idebenone (hydroxydecyl-ubiquinone). J Bioenerg Biomembr 40: 85-93.

49. Heitz FD, Erb M, Anklin C, Robay D, Pernet V, et al. (2012) Idebenone protects against retinal damage and loss of vision in a mouse model of Leber's hereditary optic neuropathy. PLoS One 7: e45182.

50. Mashima Y, Hiida Y, Oguchi Y (1992) Remission of Leber's hereditary optic neuropathy with idebenone. Lancet 340: 368-369.

51. Gueven N, Faldu D (2013) Idebenone treatment in Leber's Hereditary Optic Neuropathy (LHON): rationale and efficacy. Exp Opin Orph Drug 1: 1-9.

52. Klopstock T, Yu-Wai-Man P, Dimitriadis K, Rouleau J, Heck S, et al. (2011) A randomized placebo-controlled trial of idebenone in Leber's hereditary optic neuropathy. Brain 134: 2677-2686.

53. Klopstock T, Metz G, Yu-Wai-Man P, Büchner B, Gallenmüller C, et al. (2013) Persistence of the treatment effect of idebenone in Leber's hereditary optic neuropathy. Brain 136: e230.

54. Carelli V, La Morgia C, Valentino ML, Rizzo G, Carbonelli M, et al. (2011) Idebenone treatment in Leber's hereditary optic neuropathy. Brain 134: e188.

55. Barboni P, Valentino ML, La Morgia C, Carbonelli M, Savini G, et al. (2013) Idebenone treatment in patients with OPA1-mutant dominant optic atrophy. Brain 136: e231.

56. Bababeygy SR, Wang MY, Khaderi KR, Sadun AA (2012) Visual improvement with the use of idebenone in the treatment of Wolfram syndrome. J Neuroophthalmol 32: 386-389.

57. Kernt M, Arend N, Buerger A, Mann T, Haritoglou C, et al. (2013) Idebenone prevents human optic nerve head astrocytes from oxidative stress, apoptosis and senescence by stabilizing $\mathrm{BAX} / \mathrm{Bcl}-2$ ratio. J Glaucoma 22: 404-412.
This article was originally published in a special issue, entitled: "Nove

Treatment Strategies for Neurological and Neurodegenerative Diseases", Edited by Simon Kaja 